NTUTH-00-232

August 2000

\title{
Can nonlocal Dirac operators be topologically proper?
}

\author{
Ting-Wai Chiu \\ Department of Physics, National Taiwan University \\ Taipei, Taiwan 106, Republic of China. \\ E-mail : twchiu@phys.ntu.edu.tw
}

\begin{abstract}
By examining the analyticity of a sequence of topologically-proper lattice Dirac operators, we show that they tend to a nonlocal Dirac operator. This implies that a nonlocal lattice Dirac operator can have exact zero modes satisfying the Atiyah-Singer index theorem, in gauge backgrounds with nonzero topological charge.
\end{abstract}

PACS numbers: 11.15.Ha, 11.30.Rd, 11.30.Fs 
It is well known that all fundamental interactions are local. Therefore, it is natural to require that any lattice ( nonperturbative) formulation of these theories is local in the continuum limit. Although it is straightforward to formulate bosonic fields on the lattice with ultralocal operators, it is nontrivial to formulate the Dirac fermion field on the lattice, which can retain all its vital features in the continuum. In general, a decent lattice Dirac operator $D$ is required to satisfy the following conditions :

(i) $D$ is local.

$(|| D(x, y) \| \leq c \exp (-|x-y| / l)$ with $l \sim a$; or $D(x, y)=0$ for $|x-y|$ larger than a finite distance. )

(ii) In the free fermion limit, $D$ is free of species doublings.

( The free fermion propagator $D^{-1}(p)$ has only one simple pole at the origin $p=0$ in the Brillouin zone. )

(iii) In the free fermion limit, $D$ has the correct continuum behavior.

( In the limit $a \rightarrow 0, D(p) \sim i \gamma_{\mu} p_{\mu}$ around $p=0$.)

(iv) $D$ is $\gamma_{5}$-hermitian. $\left(D^{\dagger}=\gamma_{5} D \gamma_{5}\right.$. )

(v) $D$ satisfies the Ginsparg-Wilson relation [1].

$\left(D \gamma_{5}+\gamma_{5} D=2 a D \gamma_{5} R D\right.$, where $R$ is a positive definite Hermitian operator which commutes with $\gamma_{5}$.)

However, there exists no proof that any lattice Dirac operator satisfying these five conditions must have exact zero modes satisfying the Atiyah-Singer index theorem [2], in background gauge fields with nonzero topological charge. Though one has no doubts that conditions (ii)-(v) are necessary requirements for any topologically proper $D$, it is unclear how the locality of $D$ could play any role in the index of $D$. For example, consider the chirally symmetric Dirac operator

$$
D_{c}=a^{-1} \frac{\sqrt{H_{w}^{2}}+\gamma_{5} H_{w}}{\sqrt{H_{w}^{2}}-\gamma_{5} H_{w}}
$$

where

$$
\begin{aligned}
H_{w} & =\gamma_{5}\left(\gamma_{\mu} t_{\mu}+W-\frac{1}{2 a}\right), \\
t_{\mu}(x, y) & =\frac{1}{2 a}\left[U_{\mu}(x) \delta_{x+\hat{\mu}, y}-U_{\mu}^{\dagger}(y) \delta_{x-\hat{\mu}, y}\right], \\
W(x, y) & =\frac{1}{2 a} \sum_{\mu}\left[2 \delta_{x, y}-U_{\mu}(x) \delta_{x+\hat{\mu}, y}-U_{\mu}^{\dagger}(y) \delta_{x-\hat{\mu}, y}\right],
\end{aligned}
$$




$$
\gamma_{\mu}=\left(\begin{array}{cc}
0 & \sigma_{\mu} \\
\sigma_{\mu}^{\dagger} & 0
\end{array}\right)
$$

and

$$
\sigma_{\mu} \sigma_{\nu}^{\dagger}+\sigma_{\nu} \sigma_{\mu}^{\dagger}=2 \delta_{\mu \nu}
$$

The Dirac and color indices have been suppressed in (11)-(田). It is obvious that $D_{c}$ is topologically-proper and nonlocal, in the continuum limit. However, one can transform this nonlocal $D_{c}$ into a local $D$ which has exactly the same index of $D_{c}$ [3],

$$
D=D_{c}\left(1+a D_{c}\right)^{-1}
$$

Substituting (11) into (5), we recover the Overlap Dirac operator [4, 5]

$$
D=\frac{1}{2}\left(1+\gamma_{5} \frac{H_{w}}{\sqrt{H_{w}^{2}}}\right)
$$

which is local if the gauge background is sufficiently smooth at the scale of the lattice spacing [6, 7]. Thus, the crucial point is the existence of a topologically proper Dirac operator, whether it is a nonlocal $D_{c}$ or a local $D$ really does not matter at all. We can always transform a nonlocal $D_{c}$ into a well-defined and local $D$ which has the same index of $D_{c}$. From this point of view, the locality of $D$ does not seem to play any role in the index of $D$.

In this paper, we provide a nontrivial example of $D$ which is nonlocal and topologically-proper. Unlike the chirally symmetric $D_{c}(1)$ which has poles in the nontrivial sectors, as a consequence of the chirality sum rule [8], the present example breaks the chiral symmetry according to the GW relation, thus it is well-defined in the nontrivial sectors.

Consider the sequence of topologically-proper lattice Dirac operators as proposed by Fujikawa [9]

$$
D=a^{-1}\left(\frac{1}{2}\right)^{1 /(2 k+1)} \gamma_{5}\left(\gamma_{5}+\frac{H}{\sqrt{H^{2}}}\right)^{1 /(2 k+1)}, \quad k=0,1,2, \cdots
$$

where

$$
H=\gamma_{5}\left[(-1)^{k}\left(\gamma_{\mu} t_{\mu}\right)^{2 k+1}+W^{2 k+1}-\left(m_{0} a^{-1}\right)^{2 k+1}\right], \quad 0<m_{0}<2,
$$

and $t_{\mu}$ and $W$ are defined in (3) and (4). Note that the $(2 k+1)$-th real root of the Hermitian operator $\left(\gamma_{5}+H / \sqrt{H^{2}}\right)$ is well-defined. Recall that any Hermitian operator $h$ can be diagonalized by a unitary transformation

$$
h=U \Lambda U^{\dagger},
$$


where $\Lambda$ denotes the diagonal matrix $\left\{\lambda_{1}, \lambda_{2}, \cdots\right\}$ consisting of the real eigenvalues of $h$, and $U$ the unitary matrix formed by columns of corresponding normalized eigenvectors $\left\{u_{1}, u_{2}, \cdots\right\}$. Then any integer power of $h$ can be written as

$$
h^{\alpha}=U \Lambda^{\alpha} U^{\dagger}, \quad \alpha=\text { integer } .
$$

Thus, the $(2 k+1)$-th real root of $h$ can be defined by

$$
h^{1 /(2 k+1)}=U \Lambda^{1 /(2 k+1)} U^{\dagger},
$$

where

$$
\left[\Lambda^{1 /(2 k+1)}\right]_{i j}=\delta_{i j} \operatorname{sign}\left(\lambda_{i}\right)\left|\lambda_{i}\right|^{1 /(2 k+1)} .
$$

It is obvious that $h^{1 /(2 k+1)}$ defined in (9) satisfies the relation

$$
\left(h^{1 /(2 k+1)}\right)^{2 k+1}=h .
$$

For $k=0, D$ reduces to the Overlap-Dirac operator (6). In general, $D$ satisfies the Ginsparg-Wilson relation [1]

$$
D \gamma_{5}+\gamma_{5} D=2 a D R \gamma_{5} D
$$

with

$$
R=\left(a \gamma_{5} D\right)^{2 k}, \quad k=0,1,2, \cdots
$$

where $R$ is Hermitian and commutes with $\gamma_{5}$.

The general properties of $D$ have been derived in ref. [10]. In particular, in the naive continuum limit, the index of $D$ is independent of $k$,

$$
\operatorname{index}\left[D\left(m_{0}\right)\right]=\left\{\begin{array}{lc}
\frac{(-1)^{n+1}(d-1) !}{(d-n) !(n-1) !} Q, & 2(n-1)<m_{0}<2 n \\
0, & \text { for } n=1, \cdots, d ; \\
\text { otherwise. }
\end{array}\right.
$$

where $d$ ( even integer ) is the dimensionality of the lattice, and $Q$ is the topological charge of the gauge background. For $m_{0} \in(0,2)$, the index of $D$ is equal to $Q$, hence $D$ is topologically proper.

One of the salient features of the sequence of topologically-proper Dirac operators (7) is that the amount of chiral symmetry breaking ( i.e., r.h.s. of (11) ) decreases as the order $k$ increases. However, at finite lattice spacing, the chiral symmetry breaking of $D$ cannot be zero even in the limit $k \rightarrow \infty$, since $D$ satisfies the GW relation (11). The only possibility for $D$ to break the chiral symmetry in the limit $k \rightarrow \infty$ is that $D(p)$ becomes a piecewise continuous function in the Brillouin zone, with discontinuities somewhere at $|p| \simeq a^{-1}$, 
and $\gamma_{5} D(p)+D(p) \gamma_{5}=0$ for $|p|<a^{-1}$. Since such a $D(p)$ is non-analytic at infinite number of $p$, the corresponding $D(x, y)$ must be nonlocal in the position space.

If $D(x, y)$ is nonlocal in the free fermion limit ( i.e., $U_{\mu}(x) \rightarrow 1$ ), then it must be nonlocal in any smooth gauge background. So, it suffices to examine the non-analyticity of $D(p)$ in the free fermion limit.

In the free fermion limit, the GW Dirac operator (7) in momentum space can be written as

$$
\begin{aligned}
D(p) & =D_{0}(p)+i \gamma_{\mu} D_{\mu}(p) \\
D_{0}(p) & =a^{-1}\left[\frac{1}{2}\left(1+\frac{u(p)}{N(p)}\right)\right]^{\frac{k+1}{2 k+1}} \\
D_{\mu}(p) & =a^{-1}\left[\frac{1}{2}\left(1+\frac{u(p)}{N(p)}\right)\right]^{\frac{k+1}{2 k+1}} \sqrt{\frac{1}{a^{2} t^{2}(p)}\left(\frac{N(p)-u(p)}{N(p)+u(p)}\right)} \sin \left(p_{\mu} a\right),
\end{aligned}
$$

where

$$
\begin{aligned}
u(p) & =a^{-(2 k+1)}\left(\left[\sum_{\mu}\left(1-\cos \left(p_{\mu} a\right)\right)\right]^{2 k+1}-m_{0}^{2 k+1}\right) \\
t^{2}(p) & =a^{-2} \sum_{\mu} \sin ^{2}\left(p_{\mu} a\right) \\
N(p) & =\sqrt{\left[t^{2}(p)\right]^{2 k+1}+[u(p)]^{2}} .
\end{aligned}
$$

In the following, we set

$$
m_{0}=\left(\frac{1}{2}\right)^{1 /(2 k+1)}
$$

such that $D(p)=i \gamma_{\mu} p_{\mu}$ in the naive continuum limit.

The chiral symmetry breaking in $D(p)$ is due to its scalar component $D_{0}(p)$. For $a|p| \ll 1$,

$$
\begin{aligned}
& D_{0}(p)=a^{-1}\left(a^{2} p^{2}\right)^{k+1}+\text { higher order terms } \\
& D_{\mu}(p)=p_{\mu}\left(1+O\left(a^{2} p_{\mu}^{2}\right)\right)
\end{aligned}
$$

Thus, at small momenta or in the naive continuum limit, the chiral symmetry breaking of $D_{0}(p)$ decreases as the order $k$ increases. On the other hand, at the $2^{d}-1$ corners of the Brillouin zone, $D_{0}(p)=a^{-1}$ for all $k$. Therefore, $D_{0}(p)$ must undergo a transition from zero to $a^{-1}$ as any momentum component $\left|p_{\mu}\right|$ is increased from zero to $\pi / a$. The region of transition is essentially confined between two closed concentric hypersurfaces inside the Brillouin zone. As the order $k$ gets higher, the region of transition becomes thinner and the slope of 
the transition gets steeper. In the limit $k \rightarrow \infty$, the transition occurs only at a few hypersurfaces ( i.e., the transition is composed of several step functions ) , thus renders $D_{0}(p)$ non-analytic and $D_{0}(x)$ nonlocal.

It is instructive to work out the behavior of $D_{0}(p)$ in two dimensions (i.e., $\left.p_{3}=p_{4}=0\right)$. In the limit $k \rightarrow \infty$, (15) becomes

$$
D_{0}(p)=a^{-1} \sqrt{\frac{1}{2}\left(1+\lim _{k \rightarrow \infty} \frac{u(p)}{N(p)}\right)} .
$$

On the $\left(p_{1}, p_{2}\right)$ plane, (17)-(19) become

$$
\begin{aligned}
u(p) & =a^{-(2 k+1)}\left[\left(2-c_{1}-c_{2}\right)^{2 k+1}-\frac{1}{2}\right], \\
t^{2}(p) & =a^{-2}\left(s_{1}^{2}+s_{2}^{2}\right) \\
N(p) & =\sqrt{\left[t^{2}(p)\right]^{2 k+1}+[u(p)]^{2}} .
\end{aligned}
$$

where

$$
c_{\mu}=\cos \left(p_{\mu} a\right), \quad s_{\mu}=\sin \left(p_{\mu} a\right) .
$$

Note that the factor $a^{-(2 k+1)}$ in (24) and (26) is cancelled in the ratio $u(p) / N(p)$.

First we consider $\left(p_{1}, p_{2}\right)$ inside the square ( see Fig. 1 ) bounded by the four edges : $\left|p_{1}\right|+\left|p_{2}\right|=\pi / 2 a$. It satisfies

$$
\begin{aligned}
\left|p_{1}\right|+\left|p_{2}\right| & <\pi / 2 a \\
s_{1}^{2}+s_{2}^{2} & <1, \\
2-c_{1}-c_{2} & <1 .
\end{aligned}
$$

Hence

$$
\begin{array}{r}
\lim _{k \rightarrow \infty} \frac{\left[t^{2}(p)\right]^{2 k+1}}{[u(p)]^{2}}=\frac{0}{(-1 / 2)^{2}}=0 \\
\lim _{k \rightarrow \infty} \frac{u(p)}{N(p)}=-1 .
\end{array}
$$

From (23), we have

$$
\lim _{k \rightarrow \infty} D_{0}(p)=0, \text { for }\left|p_{1}\right|+\left|p_{2}\right|<\frac{\pi}{2 a} .
$$

Next we consider $\left(p_{1}, p_{2}\right)$ lying on the edge of the square, except its four vertices $\left\{\left(\frac{\pi}{2 a}, 0\right),\left(0, \frac{\pi}{2 a}\right),\left(-\frac{\pi}{2 a}, 0\right),\left(0,-\frac{\pi}{2 a}\right)\right\}$. It satisfies

$$
\begin{aligned}
\left|p_{1}\right|+\left|p_{2}\right| & =\pi / 2 a, \\
s_{1}^{2}+s_{2}^{2} & =1, \\
2-c_{1}-c_{2} & <1 .
\end{aligned}
$$


Thus

$$
\begin{array}{r}
\lim _{k \rightarrow \infty} \frac{\left[t^{2}(p)\right]^{2 k+1}}{[u(p)]^{2}}=\frac{1}{(-1 / 2)^{2}}=4 \\
\lim _{k \rightarrow \infty} \frac{u(p)}{N(p)}=-\frac{1}{\sqrt{5}} .
\end{array}
$$

From (23), we have

$$
\lim _{k \rightarrow \infty} D_{0}(p)=a^{-1} \sqrt{\frac{1}{2}\left(1-\frac{1}{\sqrt{5}}\right)} \text {, for }\left|p_{1}\right|+\left|p_{2}\right|=\frac{\pi}{2 a},\left|p_{i}\right| \neq \frac{\pi}{2 a} .
$$

Next we consider the four vertices $\left\{\left(\frac{\pi}{2 a}, 0\right),\left(0, \frac{\pi}{2 a}\right),\left(-\frac{\pi}{2 a}, 0\right),\left(0,-\frac{\pi}{2 a}\right)\right\}$ of the square. Each one of them satisfies

$$
\begin{aligned}
s_{1}^{2}+s_{2}^{2} & =1, \\
2-c_{1}-c_{2} & =1 .
\end{aligned}
$$

Thus

$$
\lim _{k \rightarrow \infty} \frac{u(p)}{N(p)}=\frac{1 / 2}{\sqrt{1+(1 / 2)^{2}}}=\frac{1}{\sqrt{5}} .
$$

From (23), we have

$$
\lim _{k \rightarrow \infty} D_{0}(p)=a^{-1} \sqrt{\frac{1}{2}\left(1+\frac{1}{\sqrt{5}}\right)} \text {, for }\left(p_{1}, p_{2}\right)=\left\{\left( \pm \frac{\pi}{2 a}, 0\right) ;\left(0, \pm \frac{\pi}{2 a}\right)\right\} .
$$

Next we consider $\left(p_{1}, p_{2}\right)$ lying on the closed curve ( see Fig. 1 ),

$$
\left(2-c_{1}-c_{2}\right)^{2}=s_{1}^{2}+s_{2}^{2},
$$

which circumscribes the square. The equation of this closed curve can be rewritten as

$$
\left(1-c_{1}\right)^{2}+\left(1-c_{2}\right)^{2}+c_{1} c_{2}=1
$$

Except at the four vertices, each $\left(p_{1}, p_{2}\right)$ along this curve satisfies

$$
\begin{aligned}
2-c_{1}-c_{2} & >1, \\
s_{1}^{2}+s_{2}^{2} & >1 .
\end{aligned}
$$

Thus,

$$
\begin{aligned}
\lim _{k \rightarrow \infty} \frac{\left[t^{2}(p)\right]^{2 k+1}}{[u(p)]^{2}} & =1, \\
\lim _{k \rightarrow \infty} \frac{u(p)}{N(p)} & =\frac{1}{\sqrt{2}} .
\end{aligned}
$$


From (23), we have

$\lim _{k \rightarrow \infty} D_{0}(p)=a^{-1} \frac{\sqrt{2+\sqrt{2}}}{2}$, for $\left(p_{1}, p_{2}\right)$ satisfying (31) and $\left|p_{1}\right|,\left|p_{2}\right| \neq \frac{\pi}{2 a}$.

Next we consider $\left(p_{1}, p_{2}\right)$ inside the region bounded by the closed curve and the four edges of the square, except the four vertices ( see Fig. 1 ). It satisfies

$$
\begin{aligned}
\left(2-c_{1}-c_{2}\right)^{2} & <s_{1}^{2}+s_{2}^{2}, \\
s_{1}^{2}+s_{2}^{2} & >1 .
\end{aligned}
$$

Thus

$$
\begin{aligned}
\lim _{k \rightarrow \infty} \frac{[u(p)]^{2}}{\left[t^{2}(p)\right]^{2 k+1}} & =0, \\
\lim _{k \rightarrow \infty} \frac{u(p)}{N(p)} & =0 .
\end{aligned}
$$

From (23), we have

$\lim _{k \rightarrow \infty} D_{0}(p)=a^{-1} \frac{1}{\sqrt{2}}$, for $\left(1-c_{1}\right)^{2}+\left(1-c_{2}\right)^{2}+c_{1} c_{2}<1,\left|p_{1}\right|+\left|p_{2}\right|>\frac{\pi}{2 a}$.

Finally, we consider $\left(p_{1}, p_{2}\right)$ lying outside the closed curve up to the boundary of the Brillouin zone ( see Fig. 1 ). It satisfies

$$
\left(2-c_{1}-c_{2}\right)^{2}>s_{1}^{2}+s_{2}^{2}>1 \text {. }
$$

Thus

$$
\lim _{k \rightarrow \infty} \frac{\left[t^{2}(p)\right]^{2 k+1}}{[u(p)]^{2}}=\lim _{k \rightarrow \infty}\left(\frac{s_{1}^{2}+s_{2}^{2}}{\left(2-c_{1}-c_{2}\right)^{2}}\right)^{2 k+1}=0 .
$$

Then

$$
\lim _{k \rightarrow \infty} \frac{u(p)}{N(p)}=1
$$

From (23), we have

$$
\lim _{k \rightarrow \infty} D_{0}(p)=a^{-1}, \text { for }\left(1-c_{1}\right)^{2}+\left(1-c_{2}\right)^{2}+c_{1} c_{2}>1 .
$$

To summarize,

$$
\lim _{k \rightarrow \infty} D_{0}(p)= \begin{cases}0, & \left|p_{1}\right|+\left|p_{2}\right|<\frac{\pi}{2 a} ; \\ a^{-1}\left(\frac{1}{2}-\frac{\sqrt{5}}{10}\right)^{\frac{1}{2}}, & \left|p_{1}\right|+\left|p_{2}\right|=\frac{\pi}{2 a},\left|p_{1}\right| \neq \frac{\pi}{2 a},\left|p_{2}\right| \neq \frac{\pi}{2 a} \\ a^{-1}\left(\frac{1}{2}+\frac{\sqrt{5}}{10}\right)^{\frac{1}{2}}, & \left(p_{1}, p_{2}\right)=\left\{\left( \pm \frac{\pi}{2 a}, 0\right),\left(0, \pm \frac{\pi}{2 a}\right)\right\} ; \\ a^{-1} \frac{\sqrt{2}}{2}, & \left(1-c_{1}\right)^{2}+\left(1-c_{2}\right)^{2}+c_{1} c_{2}<1,\left|p_{1}\right|+\left|p_{2}\right|>\frac{\pi}{2 a} \\ a^{-1} \frac{\sqrt{2+\sqrt{2}}}{2}, & \left(1-c_{1}\right)^{2}+\left(1-c_{2}\right)^{2}+c_{1} c_{2}=1 ; \\ a^{-1}, & \left(1-c_{1}\right)^{2}+\left(1-c_{2}\right)^{2}+c_{1} c_{2}>1 .\end{cases}
$$


In short, $D_{0}(p)$ in the Brillouin zone is essentially segregated into three piecewise constant parts. It is zero inside the square, $\sqrt{2} / 2 a$ inside the region bounded by the closed curve and the four edges of the inscribed square, and $a^{-1}$ outside the closed curve.

The discontinuities of $D_{0}(p)$ at the four edges of the inscribed square ( $\left.\left|p_{1}\right|+\left|p_{2}\right|=\frac{\pi}{2 a}\right)$ is a jump from zero to $\sqrt{2} / 2 a$, while those at the closed curve $\left[\left(1-c_{1}\right)^{2}+\left(1-c_{2}\right)^{2}+c_{1} c_{2}=1\right]$ is another jump from $\sqrt{2} / 2 a$ to $a^{-1}$. At the four vertices of the inscribed square, these two step functions merge together.

By repeating above analysis, one can obtain the discontinuities of $D_{\mu}(p)$ (16) in the limit $k \rightarrow \infty$. Their locations are the same as those of $D_{0}(p)$, at the closed curve as well as at the four edges of the inscribed square.

Along the diagonals ( $\left.\left|p_{1}\right|=\left|p_{2}\right|\right)$ of the Brillouin zone,

$$
\lim _{k \rightarrow \infty} D_{\mu}(p)= \begin{cases}p_{\mu}, & \left|p_{1}\right|=\left|p_{2}\right|<\frac{\pi}{4 a} \\ \frac{1}{2 a} \sqrt{1+\frac{1}{\sqrt{5}}}, & \left|p_{1}\right|=\left|p_{2}\right|=\frac{\pi}{4 a} \\ \frac{1}{2 a}, & \frac{\pi}{4 a}<\left|p_{1}\right|=\left|p_{2}\right|<\cos ^{-1}\left(\frac{1}{3}\right) \\ \frac{1}{2 a} \sqrt{1-\frac{1}{\sqrt{2}}}, & \left|p_{1}\right|=\left|p_{2}\right|=\cos ^{-1}\left(\frac{1}{3}\right) \\ 0, & \left|p_{1}\right|=\left|p_{2}\right|>\cos ^{-1}\left(\frac{1}{3}\right)\end{cases}
$$

In general, in the limit $k \rightarrow \infty, D_{\mu}(p)=p_{\mu}$ for $|p|<\pi / 4 a$. As $|p|$ is increased further, $\left|D_{\mu}(p)\right|$ bends down and its slope becomes less than one. Then it undergoes a discontinuous transition at the edges of the square ( $\left.\left|p_{1}\right|+\left|p_{2}\right|=\pi / 2 a\right)$, finally it drops to zero discontinuously at the closed curve $\left[\left(1-c_{1}\right)^{2}+\left(1-c_{2}\right)^{2}+c_{1} c_{2}=1\right]$, and remains zero up to the boundary of the Brillouin zone.

It is straightforward to repeat above analysis in four ( and higher ) dimensions》, however, it is unnecessary for our present purpose. Since the infinite number of discontinuities of $D(p)$ in two dimensions implies that $D(p)$ also

\footnotetext{
${ }^{1}$ In $d$ dimensions, the discontinuities of $D(p)$ in the limit $k \rightarrow \infty$ are located at two concentric closed hypersurfaces. The inscribed one is

$$
\sum_{\mu} s_{\mu}^{2}=1
$$
}

while the circumscribing one is

$$
\frac{d(d-1)}{2}+\sum_{\mu} c_{\mu}\left(c_{\mu}-d\right)+\sum_{\mu>\nu} c_{\mu} c_{\nu}=0
$$

where $c_{\mu}=\cos \left(p_{\mu} a\right)$ and $s_{\mu}=\sin \left(p_{\mu} a\right)$. Their intersections are located at:

$$
d-\sum_{\mu} c_{\mu}=\sum_{\mu} s_{\mu}^{2}=1
$$


has infinite number of discontinuities in four dimensions. This renders $D(x, y)$ nonlocal in four ( and higher) dimensions as well as in two dimensions.

In this paper, we have examined the analyticity of a sequence of topologically proper lattice Dirac operators ([7). We have shown that in the limit $k \rightarrow \infty, D(p)$ has infinite number of discontinuities. This implies that $D(x, y)$ is nonlocal in the limit $k \rightarrow \infty$. On the other hand, $D(x, y)$ is local at the zeroth order ( $k=0$ ), for gauge backgrounds which are sufficiently smooth at the scale of the lattice spacing [6, 7]. Since the index of $D$ (7) is independent of the order $k$, this suggests that the locality of a lattice Dirac operator is irrelevant to its index. In other words, if a lattice Dirac operator satisfies the four conditions (ii)-(v) but always has zero index [11], then the cause may not be due to its nonlocality. 


\section{Acknowledgement}

This work was motivated after a visit to The State University of New Jersey, Rutgers. I am grateful to Herbert Neuberger for his kind hospitality, as well as many helpful discussions. I also enjoyed the discussions together with Rajamani Narayanan and Federico Berruto. I would like to thank Jim Ball and Carleton DeTar for their kind hospitality and discussions during my visit to The University of Utah. This work was supported by the National Science Council, Republic of China, under the grant number NSC89-2112-M002-079.

\section{References}

[1] P. Ginsparg, K. Wilson, Phys. Rev. D25, 2649 (1982).

[2] M.F. Atiyah, I.M. Singer, Ann. Math. 87, 596 (1968); ibid. 93, 139 (1971).

[3] T.W. Chiu, S. V. Zenkin, Phys. Rev. D 59, 074501 (1999).

[4] H. Neuberger, Phys. Lett. B 417, 141 (1998); Phys. Lett. B427, 353 (1998).

[5] R. Narayanan, H. Neuberger, Nucl. Phys. B 443, 305 (1995).

[6] P. Hernández, K. Jansen, M. Lüscher, Nucl. Phys. B 552, 363 (1999).

[7] H. Neuberger, Phys. Rev. D 61, 085015 (2000).

[8] T.W. Chiu, Phys. Rev. D58, 074511 (1998).

[9] K. Fujikawa, Nucl. Phys. B 589, 487 (2000).

[10] T.W. Chiu, Nucl. Phys. B 588, 400 (2000).

[11] T.W. Chiu, hep-lat/9911010. 


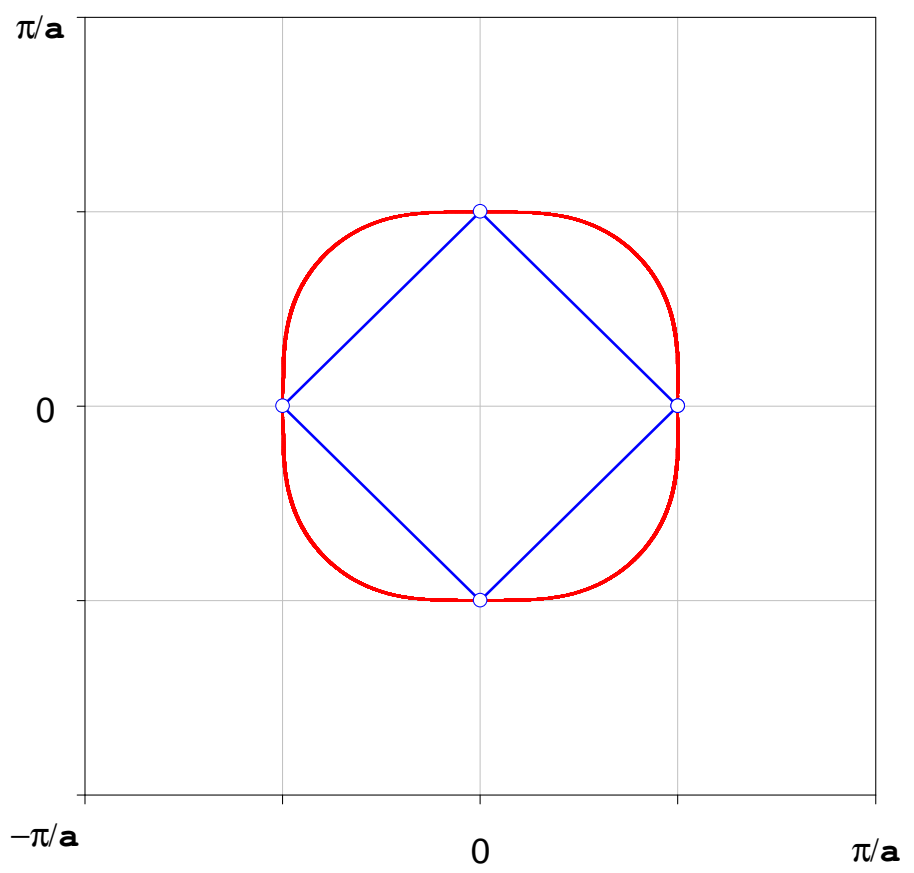

Figure 1: The discontinuities of $D_{0}(p)$ in the two-dimensional Brillouin zone. $D_{0}(p)$ is zero for $\left|p_{1}\right|+\left|p_{2}\right|<\pi / 2 a$, i.e., inside the inscribed square. Then $D_{0}(p)$ jumps from zero to $\sqrt{2} / 2 a$ at the four edges of the square, and remains $\sqrt{2} / 2 a$ for all $\left(p_{1}, p_{2}\right)$ inside the region bounded by the four edges of the square and the closed curve $\left[\left(1-\cos \left(p_{1}\right)\right)^{2}+\left(1-\cos \left(p_{2}\right)\right)^{2}+\cos \left(p_{1}\right) \cos \left(p_{2}\right)=1\right]$. Finally it jumps from $\sqrt{2} / 2 a$ to $a^{-1}$ at the closed curve, and remains $a^{-1}$ for all $\left(p_{1}, p_{2}\right)$ outside the closed curve. 